\title{
Effect of non-invasive brain stimulation on lower limb function in patients after stroke: A PRISMA-compliant systematic review and meta-analysis
}

\author{
${ }^{1}$ Xueyi Ni, ${ }^{2}$ Liru Cui, ${ }^{3}$ Ruixia Bi, ${ }^{1}$ Jinghua Qian \\ ${ }^{1}$ School of sports medicine and rehabilitation, Beijing Sport University, No. 48 Xinxi Road, Haidian \\ District, Beijing, China; ${ }^{2}$ Rehabilitation Center, Beijing Rehabilitation Hospital of Capital Medical \\ University, Beijing; ${ }^{3}$ Department of Physical Therapy, Beijing Xiaotangshan Hospital, Beijing, China.
}

\begin{abstract}
Background: In recent years, it is reported that non-invasive brain stimulation [including transcranial direct current stimulation (tDCS) and repetitive transcranial magnetic stimulation (rTMS)] could improve lower limb function in patients after stroke. However, some studies showed no effect. In the present study, we aimed to make a meta-analysis to assess effect of non-invasive brain stimulation on lower limb function in patients after stroke. Methods: Studies exploring the effect of tDCS or rTMS on lower limb function in patients after stroke were searched on the PubMed, Web of Science, EMBASE, Medline, Google Scholar before March 2021. Meta-analysis was made to summarize results of these studies. Results: The present study showed significantly better walking speed, mobility and muscle strength increase effect in tDCS group compared to sham tDCS group [walking speed: standard mean difference (SMD) $=1.14,95 \% \mathrm{CI}$ $=0.48$ to $1.80, \mathrm{I}^{2}=74.0 \%, p$ value for $\mathrm{Q}$ test $<0.001$; mobility: $\mathrm{SMD}=0.79,95 \% \mathrm{CI}=0.21$ to $1.36, \mathrm{I}^{2}$ $=53.8 \%, p$ value for $\mathrm{Q}$ test $=0.043$; muscle strength: $\mathrm{SMD}=2.79,95 \% \mathrm{CI}=0.61$ to $4.98, \mathrm{I}^{2}=93.9 \%, p$ value for $\mathrm{Q}$ test $<0.001]$. In addition, meta-analysis showed significantly better walking speed, balance and motor function increase effect in rTMS group compared to sham rTMS group [walking speed: $\mathrm{SMD}=3.31,95 \% \mathrm{CI}=1.38$ to $5.24, \mathrm{I}^{2}=92.1 \%, p$ value for $\mathrm{Q}$ test $<0.001$; balance: $\mathrm{SMD}=3.54,95 \%$ $\mathrm{CI}=1.45$ to $5.63, \mathrm{I}^{2}=95.4 \%, p$ value for $\mathrm{Q}$ test $<0.001 ;$ motor function: $\mathrm{SMD}=1.65,95 \% \mathrm{CI}=0.53$ to $2.76, \mathrm{I}^{2}=90.3 \%, p$ value for $\mathrm{Q}$ test $\left.<0.001\right]$.

Conclusions: This meta-analysis suggested that non-invasive brain stimulation improved lower limb function in patients after stroke. More large scale, blinded RCTs were necessary to confirm the effect of rTMS and tDCS on lower limb function in patients after stroke.
\end{abstract}

Keywords: Lower limb, repetitive transcranial magnetic stimulation, stroke, transcranial direct current stimulation.

\section{INTRODUCTION}

In the early stage of stroke, only approximately $20 \%$ to $30 \%$ of stroke patients showed preserved lower limb function. ${ }^{1}$ Even after spontaneous recovery, $50 \%$ of stroke patients with lower limb dysfunction still cannot walk independently. ${ }^{2}$ Rehabilitation of independent walking became one of the most important targets for rehabilitation in most stroke patients. Up to now, the effect of most lower limb rehabilitation strategies for stroke, such as electromyography biofeedback, water-based exercise, transcutaneous electrical nerve stimulation, virtual reality, on stroke is uncertain. ${ }^{3}$ According to previous studies, brain stimulation could affect brain plasticity and might be valuable for the treatment of lower limb dysfunction of stroke. ${ }^{4}$ In the recent decades, two safe, well-known, commonly used, non-invasive brain stimulation (NIBS) techniques, including transcranial direct current stimulation (tDCS) and transcranial magnetic stimulation (TMS), were widely used in rehabilitation of stroke. Increasing evidence showed that tDCS over the motor cortex, could improve walking speed and balance function in patients after stroke. ${ }^{5}$ In addition, it is reported that repetitive TMS

Address correspondence to: Jinghua Qian, School of sports medicine and rehabilitation, Beijing Sport University, No. 48 Xinxi Road, Haidian District, 102211, Beijing, China. Tel: +86-010-83052977, E-mail: JinghuaQian891273@126.com

Date of Submission: 5 June 2021; Date of Acceptance: 8 June 2021

https://doi.org/10.54029/2021asr 
(rTMS) could improve walking speed and balance function in patients after stroke. ${ }^{6}$ However, some studies showed no effect. ${ }^{78}$ In the present study, we aimed to make a meta-analysis to assess effect of non-invasive brain stimulation (including tDCS and rTMS) on lower limb function in patients after stroke.

\section{METHODS}

\section{Search strategy}

On the basis of the Preferred Reporting Items for Systematic reviews and Meta-Analysis (PRISMA) guideline ${ }^{[9]}$, a meta-analysis was used to explore the effect of non-invasive brain stimulation on lower limb function in patients after stroke. We searched for articles before March 2021 in the following databases: PubMed, Web of Science, Medline, EMBASE, Google Scholar. The following key words: ("transcranial direct current stimulation" OR " $\mathrm{tDCS}$ " OR "transcranial magnetic stimulation" OR "TMS") AND ("lower limb") AND ("stroke") were used.

\section{Inclusion criteria and exclusion criteria}

The present study included randomized controlled trials (RCTs) and crossover trails exploring effects of tDCS or rTMS on lower limb function in patients after stroke. Included studies should provide data regarding the comparison of preand post- treatment scores of walking speed, walking endurance, mobility, balance function, muscle strength and motor function of lower limb in patients after stroke. We excluded studies according to the exclusion criteria as follows: (1) studies which did not focus on non-invasive brain stimulation and lower limb function in patients after stroke; (2) reviews, meta-analyses and case studies. After that, full texts were read to exclude articles which did not provide sufficient information of pre- and post-treatment scores of walking speed, walking endurance, mobility, balance function, muscle strength or motor function of lower limb in patients after stroke.

\section{Data collection}

Data were extracted as follows: authors and publication year, research location, numbers of cases and controls, mean ages of cases and controls, gender, research type, interventions, treatment location, stimulation intensity of tDCS or frequency of rTMS, treatment intensity and time of poststroke.

\section{Meta-analysis}

Meta-analysis was conducted using STATA 12.0 software. The mean values and standard deviation (SD) of reduction or increase rate of walking speed, walking endurance, mobility, balance function, muscle strength and motor function of lower limb in patients after stroke were obtained or calculated from the included studies. The standard mean difference (SMD) and 95\% confidence intervals (CI) were computed as the effect size. We assessed heterogeneity between studies with Cochran Q test and inconsistency index $\left(\mathrm{I}^{2}\right)$ method. We used a fixed effects model when the heterogeneity was low $\left(\mathrm{I}^{2}<50 \%\right.$ or $p$ value of $Q$ test $>0.05$ ). Inversely, a random effects model was used when the heterogeneity was high $\left(\mathrm{I}^{2} \geq 50 \%\right.$ or $p$ value of $\mathrm{Q}$ test $\left.\leq 0.05\right)$. In addition, meta-regression analyses were conducted to explore source of the heterogeneity. Subgroup analyses (for different frequencies of rTMS) were conducted to explore the effect of frequency of rTMS on heterogeneities between studies. The stability of the meta-analysis was evaluated by removing 1 individual study each time. Moreover, Begg's test, Egger's test and funnel plots were used to assess publication bias.

\section{RESULTS}

\section{Search results}

Figure 1 showed the selection procedures. Supplementary Tables 1 and 2 showed study characteristics of included studies. Finally, the present study included 16 and 12 articles for $\mathrm{tDCS}^{5,7,10-23}$ and $\mathrm{rTMS}^{6,8,24-33}$, respectively. Studies for tDCS included 10 RCTs (including 110 patients after stroke given tDCS and 110 patients given sham tDCS over motor cortex) and 6 crossover trails (including 102 patients after stroke). In addition, studies for rTMS included 8 RCTs (including 130 patients after stroke given rTMS and 112 patients given sham rTMS over motor cortex) and 4 crossover trails (including 77 patients after stroke).

\section{Meta-analysis results and systematic review}

\section{Effects of tDCS on lower limb function in patients after stroke}

There were 7 studies included for effect of tDCS over the motor cortex on walking speed in patients after stroke. Meta-analysis showed significantly better walking speed increase effect in tDCS group compared to sham tDCS group with a 


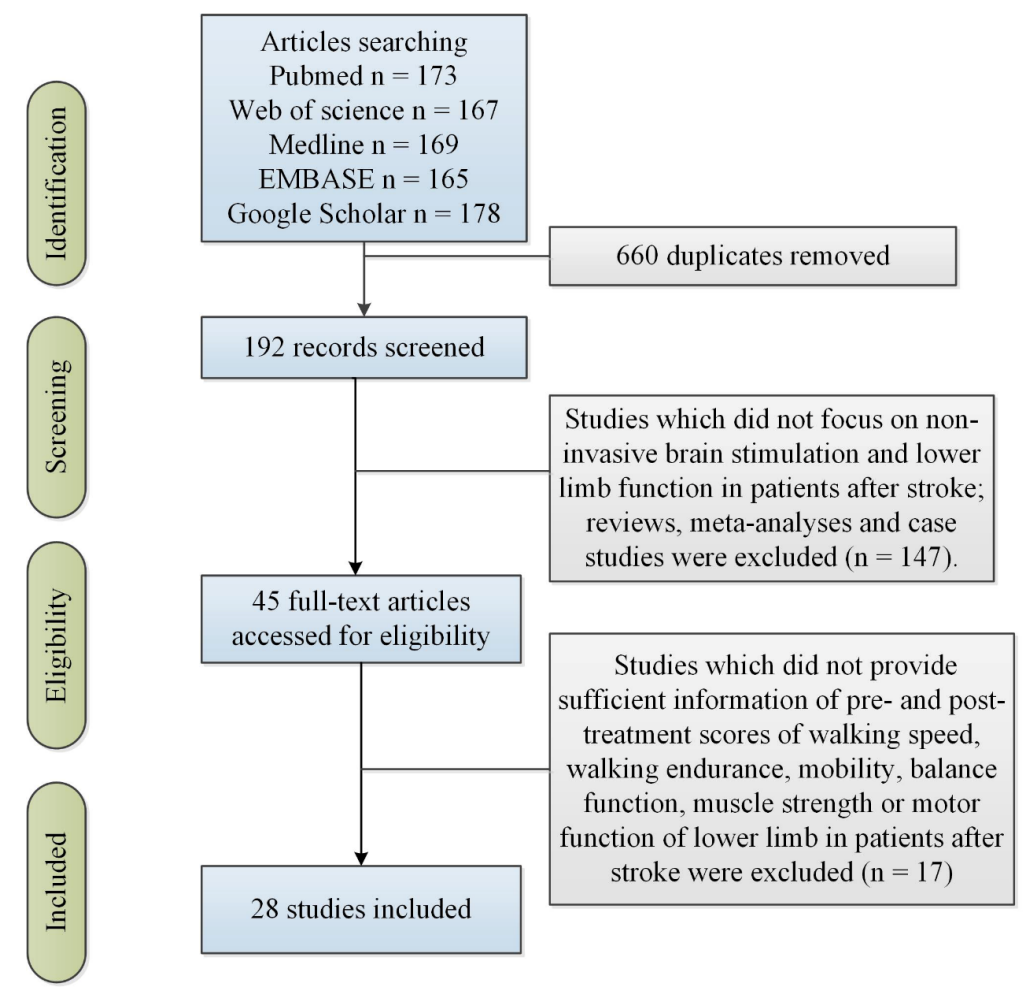

Figure 1. Characteristics of studies included in the meta-analysis.

random effect model $(\mathrm{SMD}=1.14,95 \% \mathrm{CI}=$ 0.48 to $1.80, \mathrm{I}^{2}=74.0 \%, p$ value for $\mathrm{Q}$ test $<$ 0.001 , Figure 2. A). 3 studies were included for effect of tDCS over the motor cortex on walking endurance in patients after stroke. Meta-analysis showed no significant difference in walking endurance effect in tDCS group compared to sham tDCS group with a random effect model (SMD $=-0.14,95 \% \mathrm{CI}=-1.45$ to $1.16, \mathrm{I}^{2}=79.5 \%, p$ value for $\mathrm{Q}$ test $=0.008$, Figure 2 . B). Seven studies were included for effect of tDCS over the motor cortex on mobility in patients after stroke. Meta-analysis showed significantly better mobility increase effect in tDCS group compared to sham tDCS group with a random effect model $\left(\mathrm{SMD}=0.79,95 \% \mathrm{CI}=0.21\right.$ to $1.36, \mathrm{I}^{2}=53.8 \%$, $p$ value for $\mathrm{Q}$ test $=0.043$, Figure 2. C) .5 studies were included for effect of tDCS over the motor cortex on balance in patients after stroke. Metaanalysis showed no significant difference in balance increase effect in tDCS group compared to sham tDCS group with a random effect model $\left(\mathrm{SMD}=0.51,95 \% \mathrm{CI}=-0.39\right.$ to $1.41, \mathrm{I}^{2}=83.7 \%, p$ value for $\mathrm{Q}$ test $<0.001$, Figure 2. D). Five studies were included for effect of tDCS over the motor cortex on muscle strength in patients after stroke. Meta-analysis showed significantly better muscle strength increase effect in tDCS group compared to sham tDCS group with a random effect model $\left(\mathrm{SMD}=2.79,95 \% \mathrm{CI}=0.61\right.$ to $4.98, \mathrm{I}^{2}=93.9 \%, p$ value for $\mathrm{Q}$ test $<0.001$, figure 2 . E). Four studies were included for effect of tDCS over the motor cortex on motor function in patients after stroke. Meta-analysis showed no significant difference in motor function increase effect in tDCS group compared to sham tDCS group with a random effect model $(\mathrm{SMD}=0.67,95 \% \mathrm{CI}=-0.80$ to $2.15, \mathrm{I}^{2}=89.6 \%, p$ value for $\mathrm{Q}$ test $<0.001$, figure 2.F).Additionally, meta-regression analysis showed that ages, gender, stimulation intensity and disease durations were not responsible for heterogeneity across studies regarding effects of tDCS on walking speed, mobility, balance, muscle strength and motor function in patients after stroke (Supplementary Table 3). In the present study, sensitivity analyses showed no changes in the direction of effect when any one study was excluded for all meta-analyses (see Supplementary Figure 1). Begg's test, Egger's tests and funnel plots showed no significant risks of publication bias for meta-analyses of effects of tDCS on walking speed, walking endurance, mobility, balance, muscle strength and motor function in patients after stroke (see Supplementary Table 4 and Supplementary Figure 2). 


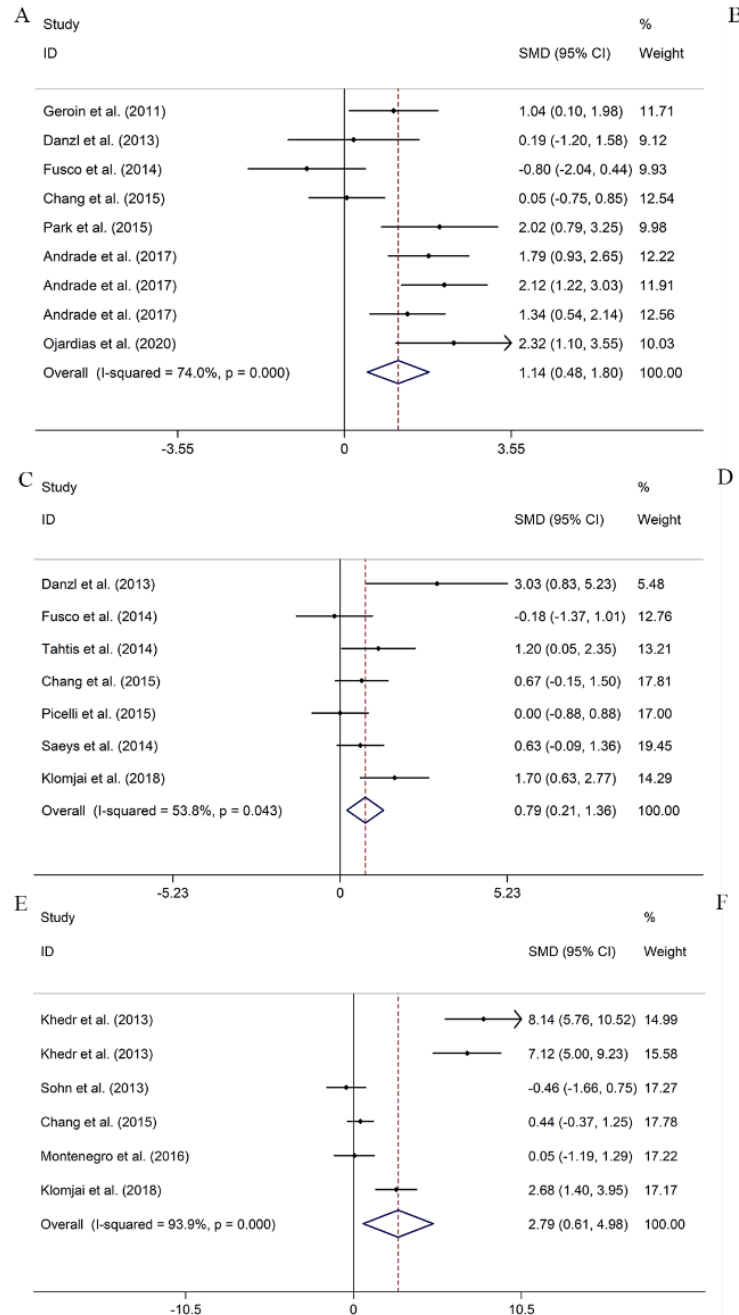

B

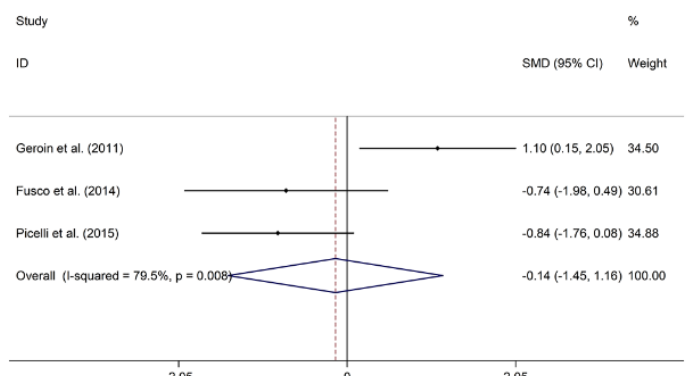

D

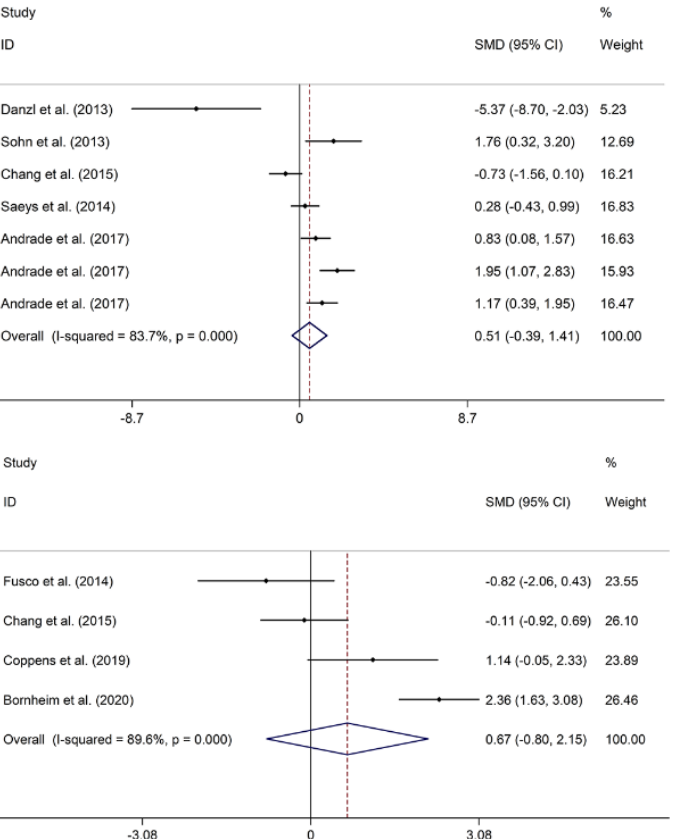

Figure 2. Forest plot regarding effects of tDCS on walking speed (A), walking endurance (B), mobility (C), balance (D), muscle strength (E) and motor function $(F)$ in patients after stroke. Abbreviation: tDCS, transcranial direct current stimulation.

\section{Effects of rTMS on lower limb function in patients after stroke}

There were 6 studies included for effect of rTMS over the motor cortex on walking speed in patients after stroke. Meta-analysis showed significantly better walking speed increase effect in rTMS group compared to sham rTMS group with a random effect model $(\mathrm{SMD}=3.31,95 \% \mathrm{CI}=1.38$ to $5.24, \mathrm{I}^{2}=92.1 \%, p$ value for $\mathrm{Q}$ test $<0.001$, figure 3. A). Subgroup study showed that HF-rTMS showed significantly better walking speed increase effect compared to sham rTMS group (SMD = $4.80,95 \% \mathrm{CI}=2.97$ to $6.63, \mathrm{I}^{2}=71.4 \%, p$ value for Q test $=0.015$; Supplementary Figure 3. A). Five studies were included for effect of rTMS over the motor cortex on balance in patients after stroke. Meta-analysis showed significantly better balance increase effect in rTMS group compared to sham rTMS group with a random effect model $\left(\mathrm{SMD}=3.54,95 \% \mathrm{CI}=1.45\right.$ to $5.63, \mathrm{I}^{2}=95.4 \%$, $p$ value for $\mathrm{Q}$ test $<0.001$, Figure 3. B). Subgroup study showed that LF-rTMS showed significantly better balance increase effect compared to sham rTMS group $(\mathrm{SMD}=2.01,95 \% \mathrm{CI}=0.28$ to $3.73, \mathrm{I}^{2}=93.1 \%, p$ value for $\mathrm{Q}$ test $<0.001$; Supplementary Figure 3. B). Eight studies were included for effect of rTMS over the motor cortex on motor function in patients after stroke. Meta-analysis showed significantly better motor function increase effect in rTMS group compared to sham rTMS group with a random effect model $\left(\mathrm{SMD}=1.65,95 \% \mathrm{CI}=0.53\right.$ to $2.76, \mathrm{I}^{2}=90.3 \%$, $p$ value for $\mathrm{Q}$ test $<0.001$, Figure 3. C). Subgroup study showed that LF-rTMS showed significantly 

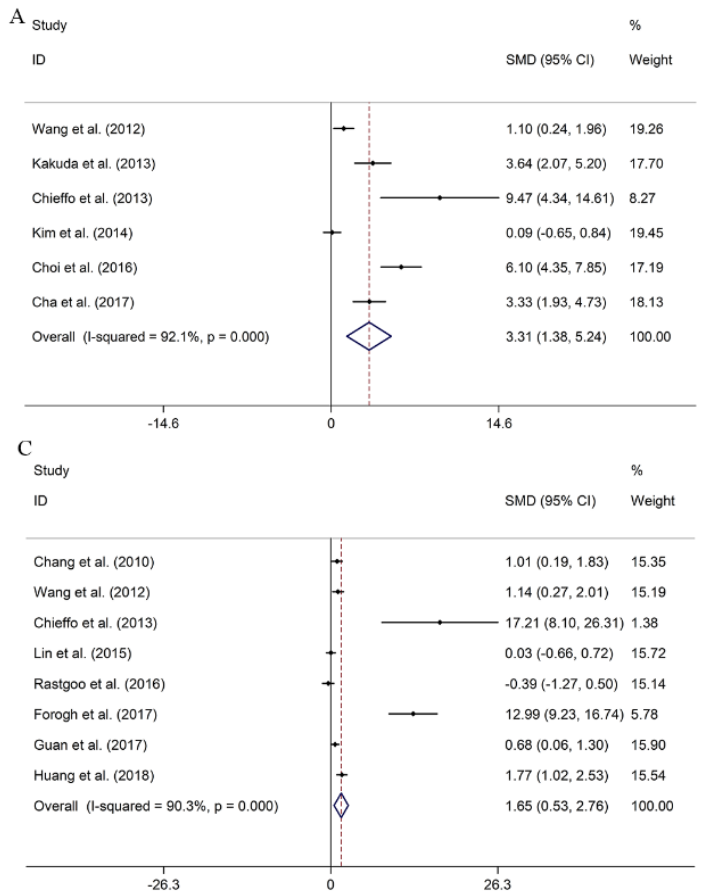

B

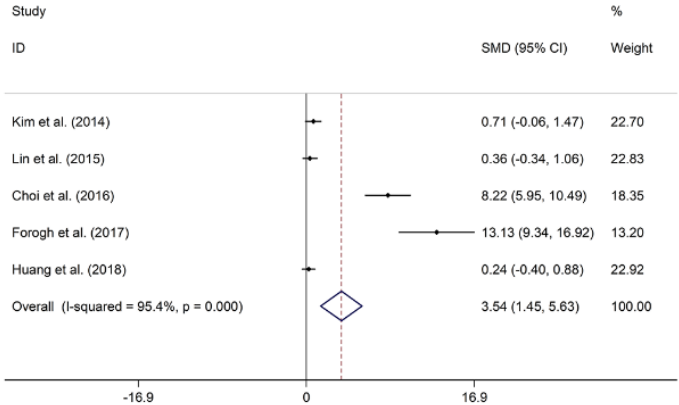

Figure 3. Forest plot regarding effects of rTMS on walking speed (A), balance (B) and motor function (C) in patients after stroke. Abbreviation: rTMS, repetitive transcranial magnetic stimulation.

better motor function increase effect compared to sham rTMS group, whereas no significant difference in motor function increase effect was showed between HF-rTMS group and sham rTMS group (LF-rTMS: SMD $=1.94,95 \%$ CI $=0.30$ to $3.58, \mathrm{I}^{2}=93.2 \%, p$ value for $\mathrm{Q}$ test $<$ $0.001 ;$ HF-rTMS: $\mathrm{SMD}=1.39,95 \% \mathrm{CI}=-0.34$ to $3.12, \mathrm{I}^{2}=84.4 \%, p$ value for $\mathrm{Q}$ test $=0.002$; Supplementary Figure 3. C). Additionally, metaregression analysis showed that ages, gender, stimulation intensity and disease durations were not responsible for heterogeneity across studies regarding effects of rTMS on walking speed, balance and motor function in patients after stroke (Supplementary Table 5). In the present study, sensitivity analyses showed no changes in the direction of effect when any one study was excluded for all meta-analyses (see Supplementary Figure 4). Begg's test, Egger's tests and funnel plots showed no significant risks of publication bias for meta-analyses of effects of rTMS on walking speed, balance and motor function in patients after stroke (see Supplementary Table 6 and Supplementary Figure 5).

\section{DISCUSSION}

The present study showed significantly better walking speed, mobility and muscle strength increase effect in tDCS group compared to sham
tDCS group. In addition, meta-analysis showed significantly better walking speed, balance and motor function increase effect in rTMS group compared to sham rTMS group.

The present study showed better walking speed, mobility and muscle strength increase effect in tDCS group compared to sham tDCS group, whereas no significant difference was showed in walking endurance, balance and motor function effect in tDCS group compared to sham tDCS group in stroke. A recent meta-analysis showed statistically significant effects in favour of tDCS for mobility and muscle strength, but no significant effects were found for improving walking speed, walking endurance and balance function. ${ }^{34}$ The present study is an updated study for the previous study. Additionally, the present study computed the mean values and SD of reduction or increase rate from baseline to follow-up time of lower limb function, whereas the previous meta-analysis computed the mean values and SD of lower limb function at the follow-up time. The computation of reduction or increase rate is more scientific. Another meta-analysis including 4 trails examined the effects of tDCS on lower limb function after stroke and showed no statistical significance associated with lower extremity function (SMD $0.2,95 \%$ CI: -1.26 to 1.67$).{ }^{35}$ Thus, more welldesigned, larger sample sized RCTs were essential 
for the investigation of effect of tDCS on lower limb function in stroke patients. In addition, the present study showed that ages, gender, stimulation intensity and disease durations were not responsible on heterogeneity across studies regarding effects of tDCS on walking speed, mobility, balance, muscle strength and motor function in patients after stroke. Bornheim et al..$^{22}$ indicated that if tDCS was applied in the acute stages of stroke, functional recovery is improved. Danzl et al. ${ }^{11}$ indicated that tDCS has the potential to enhance the effectiveness of gait training in chronic stroke. Results of these studies and meta-regression analysis suggested the significant effect of tDCS on lower limb function in both acute and chronic stroke.

In addition, the present meta-analysis indicated significantly better walking speed, balance and motor function increase effect in rTMS group compared to sham rTMS group. A recent metaanalysis indicated the effects of rTMS on lower limb function especially on motor function in patients poststroke..$^{36}$ The present study is also an updated study for the previous study. ${ }^{36}$ The previous meta-analysis computed the mean values and SD of lower limb function at the follow-up time. But our study computed the mean values and SD of reduction or increase rate from baseline to follow-up time of lower limb function. Additionally, the study aimed to explore the effect of rTMS frequency on heterogeneity across studies. Previous studies supported that rTMS works by modulating cortical excitability. HF-rTMS (> 1 Hz) facilitates cortical excitability, whereas LF- rTMS $(\leq 1 \mathrm{~Hz})$ inhibits cortical excitability. ${ }^{37}$ Subgroup study showed that HF-rTMS showed significantly better walking speed increase effect compared to sham rTMS group. Subgroup study showed that LF-rTMS showed significantly better balance and motor function increase effect compared to sham rTMS group. The meta-analysis supported that rTMS with different frequency is effective for different limb function. Thus, individualized strategy with rTMS is essential for stroke patients. In addition, the present meta-regression analysis showed that ages, gender, stimulation intensity and disease durations were not responsible for heterogeneity across studies regarding effects of rTMS on walking speed, balance and motor function in patients after stroke. Guan et al. ${ }^{32}$ showed that rTMS facilitates motor recovery of acute stroke patients. Chang et al. ${ }^{24}$ showed positive long-term effects of HF-rTMS on motor recovery during the subacute period of stroke.
Choi et al. ${ }^{6}$ indicated that HF-rTMS improves balance function in the chronic stroke patients. Thus, rTMS could be used in different periods of stroke.

Some limitations were showed in the study. Firstly, the number of included studies was limited to explore the effect of rTMS on the walking endurance, mobility and muscle strength in patients after stroke. Secondly, the number of included studies was limited to explore the sources of heterogeneities across studies regarding the effect of tDCS on walking endurance in patients after stroke.

In conclusion, the present meta-analysis suggested that non-invasive brain stimulation improved lower limb function in patients after stroke. More large scale, blinded RCTs were essential to explore the effect of rTMS and tDCS on lower limb function in patients after stroke.

\section{DISCLOSURE}

Financial support: None

Conflict of interest: None

\section{REFERENCES}

1. Van de Port IG, Kwakkel G, Van Wijk I, et al. Susceptibility to deterioration of mobility long-term after stroke: a prospective cohort study. Stroke 2006; 37(1):167-71.

2. Hankey GJ, Spiesser J, Hakimi Z, et al. Rate, degree, and predictors of recovery from disability following ischemic stroke. Neurology 2007; 68(19): 1583-7.

3. Winstein CJ, Stein J, Arena R, et al. Guidelines for adult stroke rehabilitation and recovery: A guideline for healthcare professionals from the American Heart Association/American Stroke Association. Stroke 2016; 47(6): e98-e169.

4. Coleman ER, Moudgal R, Lang K, et al. Early rehabilitation after stroke: a narrative review. Curr Atheroscler Rep 2017; 19(12):59.

5. Andrade SM, Ferreira JJA. Effects of different montages of transcranial direct current stimulation on the risk of falls and lower limb function after stroke. Neurol Res 2017; 39(12): 1037-43.

6. Choi CM, Kim JH. Effects of repetitive transcranial magnetic stimulation over trunk motor spot on balance function in stroke patients. Ann Rehabil Med 2016; 40(5): 826-34.

7. Fusco A, Assenza F, Iosa M. The ineffective role of cathodal tDCS in enhancing the functional motor outcomes in early phase of stroke rehabilitation: an experimental trial. Biomed Res Int 2014; 2014(547290.

8. Kim WS, Jung SH, Oh MK, et al. Effect of repetitive transcranial magnetic stimulation over the cerebellum on patients with ataxia after posterior circulation stroke: A pilot study. J Rehabil Med 2014; 46(5): 418-23. 
9. Moher D, Liberati A, Tetzlaff J, et al. Preferred reporting items for systematic reviews and metaanalyses: the PRISMA statement. BMJ 2009; 339(b2535

10. Geroin C, Pigelli A, Munari D, et al. Combined transcranial direct current stimulation and robotassisted gait training in patients with chronic stroke: a preliminary comparison. Clin Rehabil 2011; 25(6):537-48.

11. Danzi MM, Chelette $\mathrm{KC}$, Lee $\mathrm{K}$, et al. Brain stimulation paired with novel locomotor training with robotic gait orthosis in chronic stroke: a feasibility study. NeuroRehabilitation 2013; 33(1): 67-76.

12. Khedr EM, Shawky OA, El-Hammady DH, et al. Effect of anodal versus cathodal transcranial direct current stimulation on stroke rehabilitation: a pilot randomized controlled trial. Neurorehabil Neural Repair 2013; 27(7): 592-601.

13. Sohn MK, Jee SJ, Kim YW. Effect of transcranial direct current stimulation on postural stability and lower extremity strength in hemiplegic stroke patients. Ann Rehabil Med 2013; 37(6): 759-65.

14. Tahtis V, Kaski D, Seemungal BM. The effect of single session bi-cephalic transcranial direct current stimulation on gait performance in sub-acute stroke: A pilot study. Restor Neurol Neurosci 2014; 32(4): 527-32.

15. Chang MC, Kim DY, Park DH. Enhancement of cortical excitability and lower limb motor function in patients with stroke by transcranial direct current stimulation. Brain Stimul 2015; 8(3): 561-6.

16. Park SD, Kim JY, Song HS. Effect of application of transcranial direct current stimulation during task-related training on gait ability of patients with stroke. J Phys Ther Sci 2015; 27(3): 623-5.

17. Picelli A, Chemello E, Castellazzi P, et al. Combined effects of transcranial direct current stimulation (tDCS) and transcutaneous spinal direct current stimulation (tsDCS) on robot-assisted gait training in patients with chronic stroke: A pilot, double blind, randomized controlled trial. Restor Neurol Neurosci 2015; 33(3): 357-68.

18. Saeys W, Vereeck L, Lafosse C, et al. Transcranial direct current stimulation in the recovery of postural control after stroke: a pilot study. Disabil Rehabil 2015; 37(20): 1857-63.

19. Montenegro RA, Midgley A, Massaferri R, et al. Bihemispheric motor cortex transcranial direct current stimulation improves force steadiness in poststroke hemiparetic patients: A randomized crossover controlled trial. Front Hum Neurosci 2016; 10:426.

20. Klomjai W, Aneksan B, Pheugphrarattanatrai A, et al. Effect of single-session dual-tDCS before physical therapy on lower-limb performance in subacute stroke patients: A randomized sham-controlled crossover study. Ann Phys Rehabil Med 2018; 61(5): 286-91.

21. Coppens MJM, Staring WHA, Nonnekes J, et al. Offline effects of transcranial direct current stimulation on reaction times of lower extremity movements in people after stroke: a pilot cross-over study. J Neuroeng Rehabil 2019, 16(1): 136.

22. Bornheim S, Croisier JL, Maquet $\mathrm{P}$, et al. Transcranial direct current stimulation associated with physicaltherapy in acute stroke patients - A randomized, triple blind, sham-controlled study. Brain Stimul 2020; 13(2): 329-36.

23. Ojardias E, Aze OD, Luneau D, et al. The effects of anodal transcranial direct current stimulation on the walking performance of chronic hemiplegic patients. Neuromodulation 2020; 23(3): 373-9.

24. Chang WH, Kim YH, Bang OY, et al. Long-term effects of rTMS on motor recovery in patients after subacute stroke. J Rehabil Med 2010; 42(8): 758-64.

25. Wang RY, Tseng HY, Liao KK, et al.rTMS combined with task-oriented training to improve symmetry of interhemispheric corticomotor excitability and gait performance after stroke: a randomized trial. Neurorehabil Neural Repair 2012; 26(3): 222-30.

26. Kakuda W, Abo M, Nakayama Y, et al. Highfrequency rTMS using a double cone coil for gait disturbance. Acta Neurol Scand 2013, 128(2): 100-6.

27. Chiffo R, De Prezzo S, Houdayer E, et al. Deep repetitive transcranial magnetic stimulation with $\mathrm{H}$-coil on lower limb motor function in chronic stroke: a pilot study [J]. Arch Phys Med Rehabil 2014; 95(6): 1141-7.

28. Lin YN, Hu CJ, Chi JY, et al. Effects of repetitive transcranial magnetic stimulation of the unaffected hemisphere leg motor area in patients with subacute stroke and substantial leg impairment: A pilot study. J Rehabil Med 2015; 47(4): 305-10.

29. Rastgoo M, Nagdhi S, Nakhostin Ansari N, et al. Effects of repetitive transcranial magnetic stimulation on lower extremity spasticity and motor function in stroke patients. Disabil Rehabil 2016; 38(19): 191826.

30. Cha HG, Kim MK. Effects of strengthening exercise integrated repetitive transcranial magnetic stimulation on motor function recovery in subacute stroke patients: A randomized controlled trial. Technol Health Care 2017; 25(3): 521-9.

31. Forogh B, Ahadi T, Nazari M, et al. The effect of repetitive transcranial magnetic stimulation on postural stability after acute stroke: A clinical trial. Basic Clin Neurosci 2017; 8(5): 405-11.

32. Guan YZ, Li J, Zhang XW, et al. Effectiveness of repetitive transcranial magnetic stimulation (rTMS) after acute stroke: A one-year longitudinal randomized trial. CNS Neurosci Ther 2017; 23(12): 940-6.

33. Huang YZ, Lin LF, Chang KH, et al. Priming with $1-\mathrm{Hz}$ repetitive transcranial magnetic stimulation over contralesional leg motor cortex does not increase the rate of regaining ambulation within 3 months of stroke: A randomized controlled trial. Am J Phys Med Rehabil 2018; 97(5): 339-45.

34. Li Y, Fan J, Yang J, et al. Effects of transcranial direct current stimulation on walking ability after stroke: A systematic review and meta-analysis. Restor Neurol Neurosci 2018, 36(1): 59-71.

35. Elsner B, Kugler J, Pohl M, et al. Transcranial direct current stimulation (tDCS) for improving activities of daily living, and physical and cognitive functioning, in people after stroke. Cochrane Database Syst Rev 2016; 3(3): Cd009645.

36. Ghayour-Najafabadi M, Memari AH, Hosseini L, et 
al. Repetitive transcranial magnetic stimulation for the treatment of lower limb dysfunction in patients poststroke: A systematic review with meta-analysis. J Stroke Cerebrov Dis 2019; 28(12): 104412.

37. Sasaki N, Kakuda W, Abo M. Bilateral high- and low-frequency rTMS in acute stroke patients with hemiparesis: A comparative study with unilateral high-frequency rTMS. Brain Inj 2014; 28(13-14): 1682. 